EPJ Web of Conferences 57, 03002 (2013)

DOI: $10.1051 /$ epjconf/20135703002

(C) Owned by the authors, published by EDP Sciences, 2013

\title{
Towards hybrid quantum systems: Trapping a single atom near a nanoscale solid-state structure
}

\author{
T.G. Tiecke ${ }^{1,2, a, b}$, J.D. Thompson ${ }^{1, b}$, J. Feist ${ }^{1,3}$, A. Akimov ${ }^{4}$, A. Zibrov ${ }^{1}$, \\ V. Vuletić ${ }^{2}$ and M.D. Lukin ${ }^{1}$ \\ ${ }^{1}$ Department of Physics, Harvard University, Cambridge, MA 02138, USA \\ ${ }^{2}$ MIT-Harvard Center for Ultracold Atoms, and Research Laboratory of Electronics, MIT, \\ Cambridge, Massachusetts 02139, USA \\ 3ITAMP, Harvard-Smithsonian Center for Astrophysics, Cambridge, Massachusetts 02138, \\ USA \\ ${ }^{4}$ Russian Quantum Center and Lebedev Institute of Physics, Moscow, Russia
}

\begin{abstract}
We describe and demonstrate a method to deterministically trap single atoms near nanoscale solid-state objects. The trap is formed by the interference of an optical tweezer and its reflection from the nano object, creating a one-dimensional optical lattice where the first lattice site is at $z_{0} \sim \lambda / 4$ from the surface. Using a tapered optical fiber as the nanoscopic object, we characterize the loading into different lattice sites by means of the AC-Stark shift induced by a guided fiber mode. We demonstrate a loading efficiency of 94(6)\% into the first lattice site, and measure the cooperativity for the emission of the atom into the guided mode of the nanofiber. We show that by tailoring the dimensions of the nanofiber the distance of the trap to the surface can be adjusted. This method is applicable to a large variety of nanostructures and represents a promising starting point for interfacing single atoms with arbitrary nanoscale solid-state systems.
\end{abstract}

\section{INTRODUCTION}

The development of hybrid quantum systems is an active field of research [1-3]. Hybrid quantum systems aim to address the conflicting challenges of simultaneous isolation and control of quantum systems by combining two or more systems while preserving their respective advantages. Integrating single atoms with microscopic and nanoscopic objects is of particular interest due to the favorable properties of both systems. On one hand neutral atoms have excellent coherence properties and trapping them close to surfaces is less challenging than with ions [4]. On the other hand nanoscale structures promise strong interactions, scalability and potential applications. The combination of single atoms and nanotechnology allows engineering of strongly interacting systems while maintaining the excellent quantum control of neutral atoms.

To integrate atoms with solid-state systems a crucial challenge is to trap atoms at distances very close to the surface. In particular, in order to realize an efficient atom-photon interface the atom has to be trapped at subwavelength distances from the nanophotonic structure. Trapping at these distances is challenging due to large short-range attractive surface forces (Casimir-Polder and van der Waals) which have to be overcome. Traps for ultra-cold atoms near surfaces have been an active research

\footnotetext{
a e-mail: tiecke@physics.harvard.edu

b These authors contributed equally to this work.
}

This is an Open Access article distributed under the terms of the Creative Commons Attribution License 2.0, which permits unrestricted use, distribution, and reproduction in any medium, provided the original work is properly cited. 


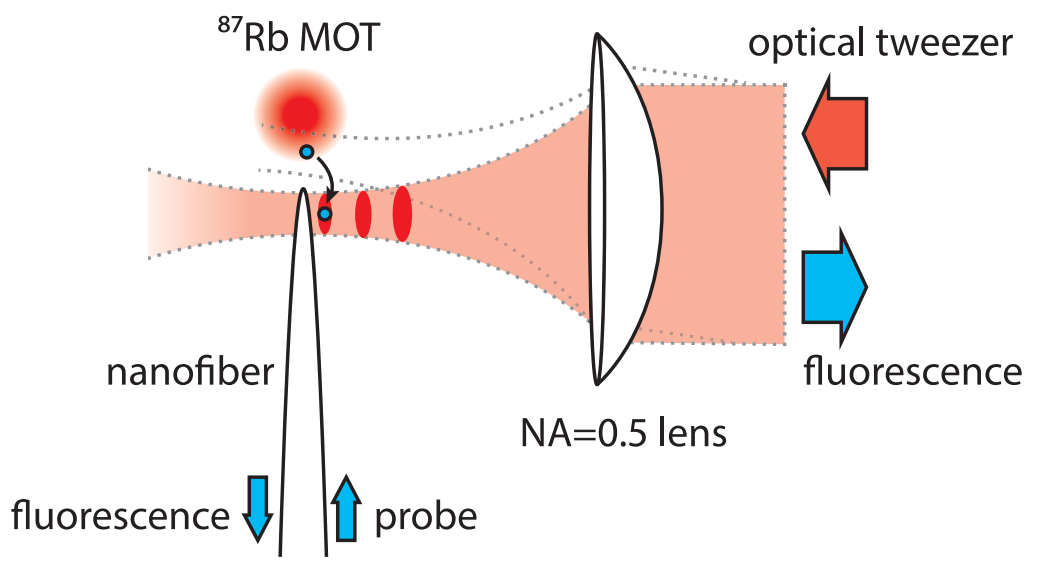

Figure 1. Trapping a single atom near a nanofiber. The trap is formed by the interference of an optical tweezer and the reflection from the nanofiber. The optical tweezer is focused by a high numerical aperture $(\mathrm{NA}=0.5)$ lens to a focus with a waist of $w_{0}=0.9 \mu \mathrm{m}$. The one-dimensional optical lattice has a local minimum at $z_{0} \sim \lambda / 4$ from the surface of the nanofiber and additional minima spaced by $\lambda / 2$. A single ${ }^{87} \mathrm{Rb}$ atom is loaded from a MOT at $\sim 20 \mu \mathrm{m}$ from the nanofiber in the optical tweezer and transported into the lattice by scanning the tweezer over the nanofiber. Fluorescence of the atom is collected using single-photon counting modules (SPCMs) through the lens or through the nanofiber. For the measurements in figure 3 a probe beam is sent through the nanofiber.

topic for many years. Atom-chip [5] methods using magnetic traps generated by patterned electrodes have reached distances down to $500 \mathrm{~nm}[6,7]$ using a Bose-Einstein condensate, at which point surface forces [8] become too strong compared to the achievable magnetic trapping forces. Optical dipole traps can be much tighter, and trapping methods based on the steep gradients of evanescent optical fields have been studied extensively [9-11], and have gained interest recently in the context of nanofibers [12-14]. In these experiments a disordered ensemble of atoms is trapped at a distance down to $230 \mathrm{~nm}$ from a nanofiber. Optical traps based on lattices induced by reflections from the surface have been demonstrated to confine atoms within $1.5 \mu \mathrm{m}$ of a surface [15-17]. Lastly, experiments at distances down to $100 \mathrm{~nm}$ have been conducted using untrapped, transiting atoms in the context of surface force measurements [18] and strong-coupling to an optical mode of a microtoroid resonator [19]. To realize an atom-photon interface which is intrinsically scalable a method to deterministically trap single atoms at a subwavelength distance from the surface is required.

Here, we describe and demonstrate a method to deterministically trap single atoms at subwavelength distances from an arbitrary nanoscale object. The trap is formed by the interference of a running wave optical tweezer with its reflection from the nanostructure. The interference pattern creates a trap at a distance of $z_{0} \sim \lambda / 4$ from the surface (henceforth called the nanotrap), and this distance can be tuned around $\lambda / 4$ by tailoring the reflection from the nanostructure. We trap single atoms near a tapered nanofiber and show near perfect loading into the nanotrap. We measure the cooperativity for emission into the guided modes of the single mode nanofiber. Finally, we discuss possible applications of this method.

\section{TRAPPING ATOMS NEAR SURFACES}

\subsection{Trap concept}

The concept of our experiment is outlined in Figure 1: we trap single atoms in a tightly focused red-detuned optical tweezer $\left(\lambda=815 \mathrm{~nm}, U_{0}=1.6 \mathrm{mK}\right)$ which is loaded from a magneto-optical trap (MOT), similar to the setup of Ref. [20]. We operate the optical tweezer in the so called collisional 
blockade regime where the number of atoms in the tweezer is conveniently limited to one or zero due to light-induced collisions [21]. The tight focus is obtained with an aspheric lens (numerical aperture $(\mathrm{NA})=0.5$ ), yielding a beam waist of $w_{0}=0.9 \mu \mathrm{m}$. The presence of an atom in the tweezer is detected by measuring the fluorescence of the atom collected through the high NA lens which is also used to create the dipole trap. After an atom is detected the MOT is switched off and we employ Raman sideband cooling [22] to decrease the thermal spread of the atom's wavefunction in the tweezer. The atom is cooled to its motional groundstate in the two radial dimensions and to an axial vibrational quantum number of $\bar{n}_{z} \simeq 8$. Subsequently, the trap is moved in $20 \mathrm{~ms}$ over a distance of $\sim 40 \mu \mathrm{m}$ to a few $\mu \mathrm{m}$ from the nanostructure by tilting a scanning galvometer mirror. The trapping and cooling has to be performed well away from the nanostructure to suppress background light of the MOT beams, scattered from the nanostructure. Subsequently, the tweezer is moved over the nanostructure, slowly increasing the amplitude of the reflected light, corresponding to a gradual turning on of the surface optical lattice. For sufficiently slow motion of the optical tweezer (tweezer velocity $<1 \mu \mathrm{m} / \mathrm{ms}$ ) the trap deformation is adiabatic. While this method is experimentally easy to implement, the absence of significant heating or loss is crucial for achieving a high loading efficiency and a scalable method for trapping single atoms near nanostructures.

The distance $z_{0}$ of the closest lattice site to the surface is determined by the phase shift of the light scattered from the surface, which for zero phase shift corresponds to $z_{0}=\lambda / 4$. Both the amplitude and phase of the reflected field depend on the details of the nanostructure and thereby influence the trap properties. Scattering of a plane wave from a dielectric cylinder is well described in the literature $[23,26]$ and has recently been studied in the context of coupling to the eigenmodes (leaky modes) of nanocylinders [27]. The scattered field is given by a sum over the contributions of the different scattered partial waves which interfere with each other and with the incoming plane wave. This interference modifies both the distance of the trap to the surface as well as the trap depth. Figure $2 \mathrm{~b}$ and $2 \mathrm{c}$ show the distance to the surface of the nanofiber and the trap depth as a function of fiber radius, calculated both using the analytical solution for a plane wave [23] and a full three-dimensional finite-difference time domain (FDTD) simulation. Both calculations include the Casimir-Polder potential which are obtained using SCUFF-EM [24].

To validate this model we perform a measurement to determine to local trap depth; we trap and cool a single atom as described above and move the atom to a position along the fiber taper axis but offset to the side (as shown in Fig. 2a). Subsequently, we load the atom in the optical lattice and transfer it back into free space again where we detect if the atom has survived the trajectory by pulsing on the MOT beams and collecting the atomic fluorescence through the high NA lens as described above. The atom is loaded in a focal plane for which we expect to load either the first or the second lattice site. The first lattice site, however, was likely unstable in this measurement due to rubidium adsorption on the fiber as will be explained below. We repeat this measurement for various positions along the nanofiber axis and measure the survival probability. The results are shown in Figure $2 \mathrm{~d}$ and show qualitative agreement with the trap depth calculations: the survival probability is proportional to the calculated trap depth. Additionally, from our model we estimate the distance to the surface can be tuned tailoring the scattering phase-shift by $\pm 60 \mathrm{~nm}$. This property is a powerful tool to tune the coupling strength of the atom to the nanostructure. The loss of trap depth in such a case can be compensated by using more laser power for the optical tweezer, within the limits of not melting the nanostructure by the highly focussed beam.

To determine the efficiency of loading an atom in the nanotrap we spectroscopically measure a spatially varying AC-Stark shift due to a guided mode in the fiber. This shift is induced by a probe beam sent into the fiber taper and tuned in between the $F=1 \rightarrow F^{\prime}=\ldots$ and $F=2 \rightarrow F^{\prime}=\ldots$ transitions on the D2-line. The fiber taper mode is exponentially confined with a typical decay length of $150 \mathrm{~nm}$ at the position along the fiber where we perform the experiment, therefore, the lattice site closest to the surface will experience a significantly larger AC-Stark shift than the second lattice site. Before loading the trap we prepare the atom in the $\left|F=2, m_{F}=0\right\rangle$ hyperfine and Zeeman sublevel 

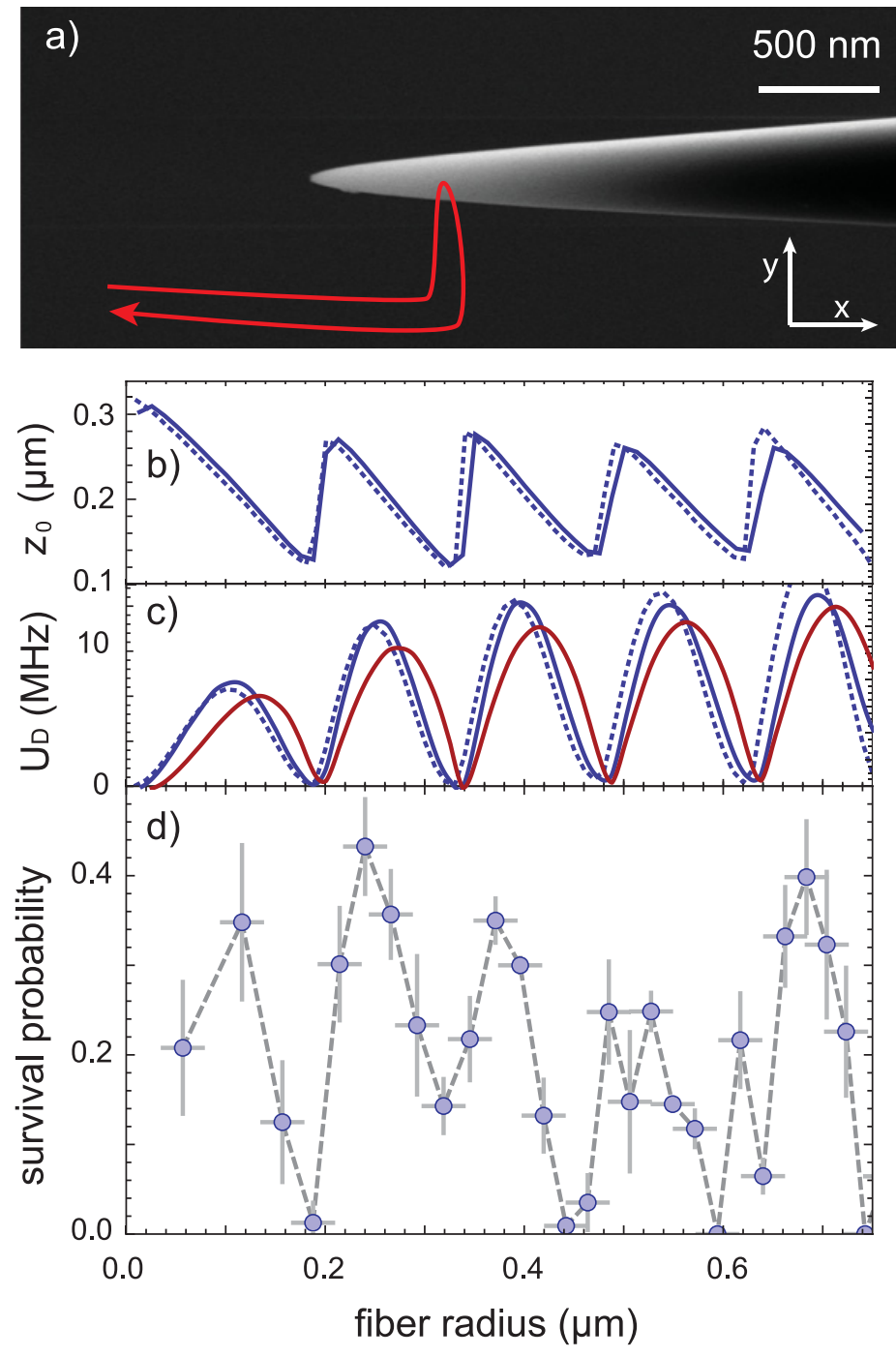

Figure 2. a.) A scanning electron microscope (SEM) image of a nanofiber similar to the device used for the presented experiments. The optical tweezer is propagating along $\hat{z}$ into the plane. The red line sketches the trajectory of the optical tweezer for loading the nanotrap. b.c.) Simulation of the the distance $z_{0}$ to the fiber surface and the trap depth $U_{D}$ for a free space trap depth $U_{0}=20 \mathrm{MHz}$ as a function of the fiber radius. For an increasing fiber radius an increasing number of partial waves contributes to the reflected light and therefore modifies the trap depth and distance to the surface. This can be used to tune the distance to the fiber surface. The solid curves are 3D finite-difference time domain (FDTD) simulations and the dotted lines are obtained from a plane wave scattered from an infinite dielectric cylinder (see text) both accounting for Casimir-Polder and van der Waals surface forces; the trap properties are very well described by the analytical solution. The blue (red) solid curves in c. are for the first (second) lattice site showing similar behavior of the trap depth. The distinct phase shift between the numerical and analytical results is due to neglecting the Gouy phase of the Gaussian focus in the plane wave description of the analytical solution. d.) survival probability of an atom following the trajectory sketched by the red line in figure a. (see text) as a function of position along the $\mathrm{x}$-axis, and therefore the fiber radius. The atom was loaded in a focal plane in between the first and second lattice site under conditions where the first lattice site was likely unstable due to rubidium adsorption (see text). The survival probability shows a qualitatively similar behavior as the trap depth in figure c. Figures b-d. are all for a tweezer polarized orthogonal to the nanofiber axis $\mathbf{E}=E_{0} \mathbf{e}_{\mathbf{y}}$. 

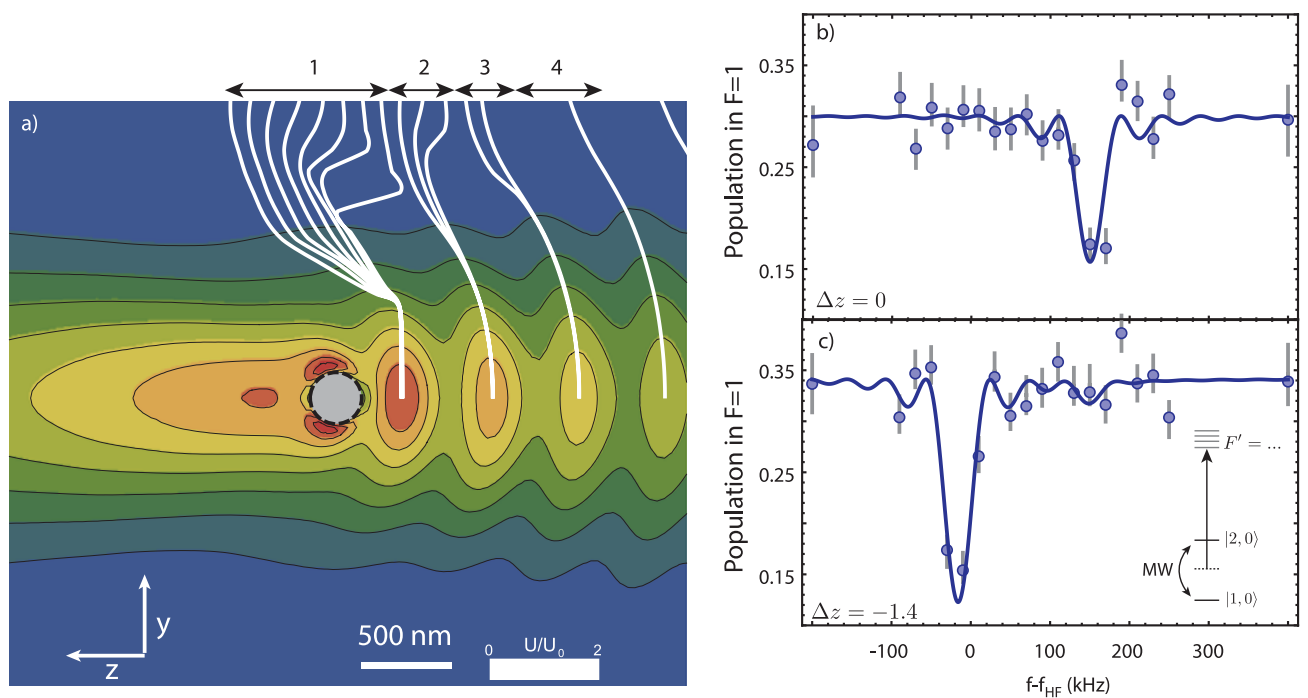

Figure 3. a.) FDTD simulation of the potential in the $y z$-plane normalized to the free space trap depth; the grey circle is the fiber cross-section. The white lines indicate the local potential minimum for the optical tweezer while it is scanned over the nanofiber. Over a range of focal planes of $\sim 0.8 \mu \mathrm{m}$ the trap minimum is translated to the first lattice site. b.-c.) Microwave spectra on the $|2,0\rangle \rightarrow|1,0\rangle$ transition probing the spatially-varying AC-Stark shift induced by an off resonant probe beam sent through the fiber. The (higher, lower) frequency peaks show the atoms loaded into the (first, second and higher) lattice sites. The focal plane of the tweezer is in focus with the nanofiber for b. and defocused by $\Delta z=-1.4 \mu \mathrm{m}$ for c. From this data we extract (see text) a loading efficiency of 94(6)\% in the lattice of which $100_{-12}^{+0} \%$ is in the first lattice site.

by means of optical pumping and coherent transfer. Subsequently, we load the lattice by scanning the tweezer over the nanofiber at a fixed focal plane. Once the lattice is loaded we drive a microwave $\pi$-pulse to the $\left|F, m_{F}\right\rangle=|1,0\rangle$ state and detect the population in the $F=1$ manifold by heating atoms in the $F=2$ manifold out of the trap with resonant light followed by fluorescence detection of the remaining atoms. To load the different lattice sites we modify the focal plane of the dipole trap before loading the lattice. The results are shown in figure 3. Accounting for independently measured depolarization due to scattering from the dipole trap and losses from background gas collisions we obtain a loading efficiency of $94(6) \%$ in the lattice of which $100_{-12}^{+0} \%$ is in the first lattice site. These results are consistent with lossless loading of the nanotrap. Finally, we measure the lifetime of the atom in the nanotrap to be $\tau=150(20) \mathrm{ms}$.

The results discussed above show the discrete nature of the optical lattice, however, since accurately determining the absolute power of the probe beam at the fiber tip is challenging and the exponential nature of the confined mode only reveals the relative distance to the fiber surface this measurement is not conclusive for trapping in the lattice site closest to the surface. To determine the absolute distance to the surface we perform an experiment where we drive the atom from the side with a beam resonant with the $F=2 \rightarrow F^{\prime}=3$ transition. We drive the atom well above saturation, such that (in the limit of weak coupling to the nanostructure) the photon scattering rate is known to be $\Gamma_{0}=\gamma / 2$, where $\gamma=$ $2 \pi \times 6 \mathrm{MHz}$ is the natural linewidth. We collect the photons emitted into the fiber mode with a single photon counting module (SPCM). The ratio of emission into the guided fiber mode $\Gamma_{f}$ versus the freespace emission rate determines the cooperativity $C=\Gamma_{f} / \Gamma_{0}$, which is a strong function of the position around the fiber (see figure $4 \mathrm{a}$ ). In figure $4 \mathrm{~b}$ the numerically calculated cooperativity for coupling to the fiber mode is shown at the fiber radius of $125 \mathrm{~nm}$, indicated by the dashed line in figure $4 \mathrm{a}$. We verify the local fiber radius from a measurement as presented in figure 2 . Figure $4 \mathrm{c}$ shows a typical fluorescence collection measurement averaged over 6000 loading events. Due to the photon scattering 

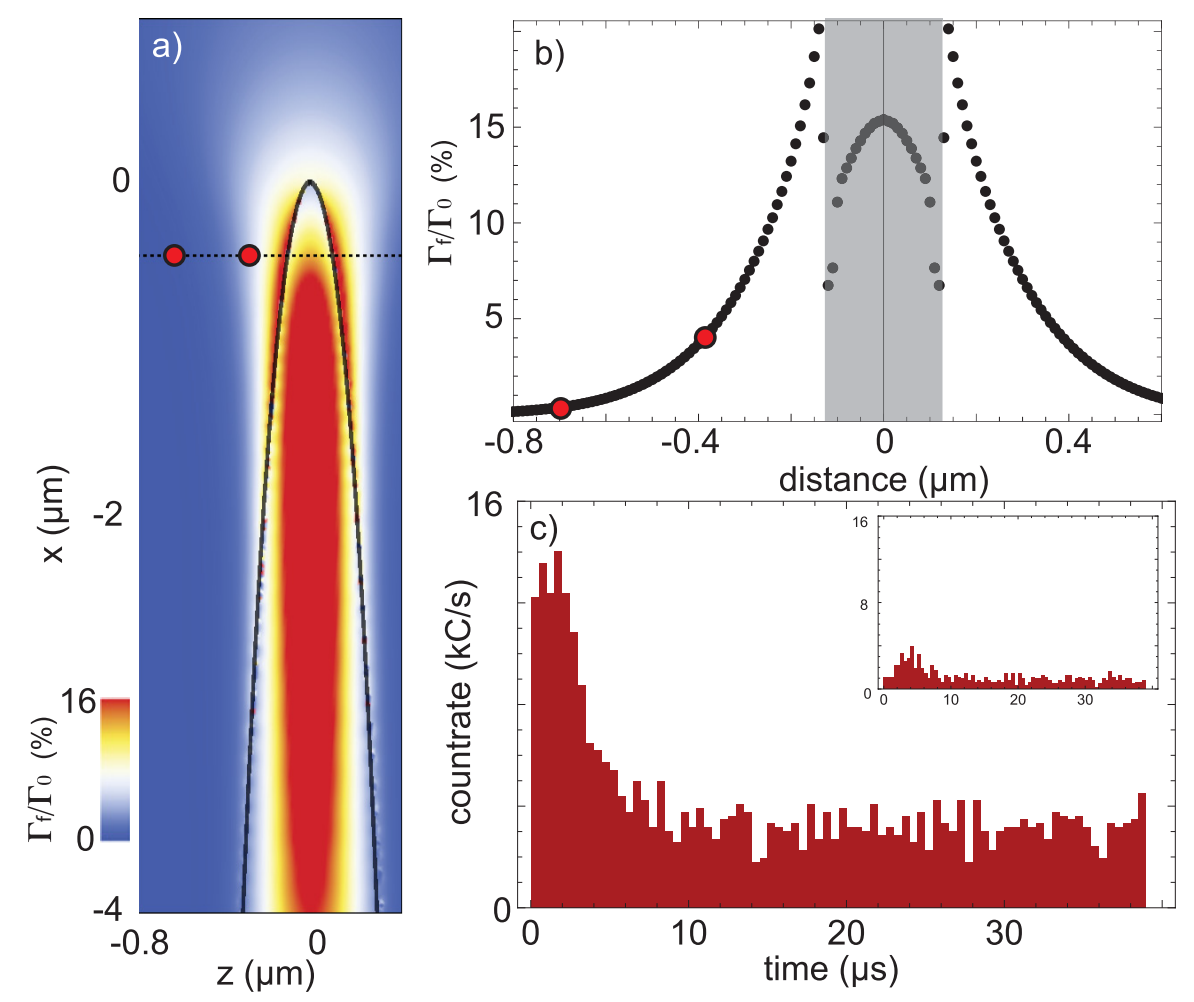

Figure 4. a.) A Boundary Element Method (BEM) simulation of the emission of the atom into the fiber. Plotted is the cooperativity of emitting into the nanofiber mode propagating towards increasing fiber radii and averaged over all atomic dipole orientations. The dashed line shows the $\mathrm{x}$-position where the atom is loaded. The two red circles indicate the position of the calculated trap positions of the first and second lattice site. b.) A cut through the data of figure a. indicated by the dotted line. The shaded gray area indicates the fiber. The two red dots indicate the position of the first and second lattice sites. c.) Photon collection through the nanofiber from an atom loaded in the first lattice site and driven above saturation. Correcting for loss (see text) we determine a cooperativity of $\Gamma_{f} / \Gamma_{0}=0.015(6)$, thereby concluding the atom is trapped in the first lattice site (see text). Inset shows the same measurement performed for an atom loaded into the second lattice site. The delayed signal agrees with Monte-Carlo simulations of the atom being heated out of the trap and having a finite probability of transiting the fiber after a few $\mu$ s.

the atom is heated out of the trap causing the decay in the fluorescence signal. To obtain the initial position of the atom before scattering photons we analyze the first $250 \mathrm{~ns}$ of the fluorescence signal during which the atom cannot have moved by more than $50 \mathrm{~nm}$ from the radiation pressure of the probe beam and thermal energy of the atom. For each atom we measure $0.9 \times 10^{-2}$ detector clicks in the first $250 \mathrm{~ns}$. Correcting for quantum efficiency of the detector, atom loss and independently measured fiber coupling efficiency we obtain a cooperativity of $C=0.015(6)$. Here, we have conservatively assumed no loss in the fiber taper, yielding an underestimate for the cooperativity. Comparing this to the numerically calculated cooperativity at the local fiber radius we obtain an upper limit for the distance to the fiber surface of $d<375 \mathrm{~nm}<\lambda / 2$, confirming that the atom is trapped in the closest lattice site.

A few additional points are worth noting. Adsorption of rubidium on a surface is known to generate patch potentials $[6,8]$ which can significantly affect the trap properties. We have observed that the nanotrap is unstable unless if we periodically (every 5 minutes for 30 seconds) apply $\sim 40 \mu \mathrm{W}$ of blue $(\lambda=472 \mathrm{~nm})$ light through the taper. We interpret this as the nanotrap being unstable due to 
patch potentials formed by adsorbed rubidium and that a stable nanotrap can be obtained if rubidium is desorbed from the surface by the blue light. Additionally, we have studied the two possibilities of loading the optical lattice; by approaching the nanofiber along its axis or orthogonal to its axis. Both methods have shown to load successfully in the optical lattice with similar performance.

Another point of consideration is the polarization of the electric field at the position of the nanotrap. Elliptical polarization of the electric field induces vector lightshifts on the Zeeman sublevels of the atomic groundstate. These lightshifts will result in different trapping potentials for the different Zeeman sublevels, leading to decoherence and fluctuating dipole force heating arising from spontaneous Raman scattering. Even for perfectly linearly polarized incoming fields, tight focusing [22] and optical fields near nanostructures [28] will generate undesirable elliptical polarized field components.

If we approximate the nanofiber as an infinite cylinder with its axis along $\mathbf{e}_{\mathbf{x}}$ and the optical tweezer as a plane wave propagating in the $y z$-plane, the system can be described in a two-dimensional geometry. For two dimensional systems the solutions to the Maxwell equations can be classified as either transverse-electric (TE), where $\mathbf{E}=E_{y} \mathbf{e}_{\mathbf{y}}+E_{z} \mathbf{e}_{\mathbf{z}}$ or transverse-magnetic (TM), where $\mathbf{E}=E_{x} \mathbf{e}_{\mathbf{x}}$. These two cases result in significantly different nanotrap polarization: for an optical tweezer propagating along the $z$-axis and polarized along the nanofiber axis $\mathbf{E}_{\mathrm{ODT}}=E_{0} \mathbf{e}_{\mathbf{x}}$, the scattering excites only TM modes which are also polarized along $\mathbf{e}_{\mathbf{x}}$. Therefore, the nanotrap will be linearly polarized. For the case where the optical dipole trap is polarized orthogonal to the nanofiber axis, $\mathbf{E}_{\mathrm{ODT}}=E_{0} \mathbf{e}_{\mathbf{y}}$, the scattering excites TE modes which are polarized in the $y z$-plane, with a phase and orientation depending on the specific properties of the nanostructure. The interference of the incoming field with the scattered field can result in a strongly elliptical polarized nanotrap. Therefore, the preferred polarization axis for the nanotrap is along the $x$-axis, which we have implemented in follow-up experiments.

\section{CONCLUSION AND OUTLOOK}

We have described and experimentally demonstrated a method to deterministically trap and manipulate single atoms at a distance of $\sim 200 \mathrm{~nm}$ from a surface. Our results are consistent with lossless loading of the nanotrap and we observe extended lifetimes $(>100 \mathrm{~ms})$. We describe how to tune the distance to the surface over $\pm 60 \mathrm{~nm}$ by tailoring the reflection from the nanostructure. The presented method is largely independent of the nanostructure near which the atom is trapped, therefore, this method is suitable to trap single atoms in the near-field of a large variety of structures. Here, we have demonstrated the method using a nanofiber, and recently we have used this method to trap a single atom in the near-field of a photonic-crystal cavity and observe coupling to the cavity [29].

The present results open exciting prospects for future studies. First, due to the strong localization of the atom near the surface this method could be used to probe atom-surface interactions at submicron lengthscales [8], possibly putting bounds on non-Newtonian gravity forces [31]. Second, this technique is intrinsically scalable and could be used to simultaneously implement multiple hybrid quantum systems thereby realizing a quantum network [33]. Arrays of multiple optical tweezers, each trapping single atoms, have been recently demonstrated [30], and such an array could be combined with our method to efficiently load multiple nanotraps coupled to either the same or different nanostructures. Alternatively, by starting from a quantum degenerate gas it might be possible to load deep subwavelength arrays of traps formed by plasmonic structures [32]. This would open up the possibility to engineer few- to many-body quantum systems at sub-wavelength length scales, controlling individual atoms and individual interactions, enabling to study strongly correlated states of ultra-cold atoms in a new regime of higher densities, stronger and longer-ranged interactions [32], approaching real-world solid-state quantum systems. 
We acknowledge funding from the NSF, CUA, DARPA, AFOSR, MURI and the Packard Foundation. JDT acknowledges support from the Fannie and John Hertz Foundation and the NSF GRFP. JF acknowledges support from the NSF through a grant to ITAMP.

\section{References}

[1] R. J. Schoelkopf, S. M. Girvin, Nature, 451 (2008), 664-669.

[2] M. Wallquist, K. Hammerer, P. Rabl, M. Lukin and P. Zoller, Physica Scripta, 2009 (2009), 014001.

[3] Philipp Treutlein, Claudiu Genes, Klemens Hammerer, Martino Poggio and Peter Rabl, arXiv:1210.4151.

[4] N. Daniilidis, S. Narayanan, S. A. Möller, R. Clark, T. E. Lee, P. J. Leek, A. Wallraff, St. Schulz, F. Schmidt-Kaler and H. Häffner, New J. Phys., 13 (2011), 013032.

[5] J. Reichel, W. Hänsel and T. W. Hänsch, Phys. Rev. Lett., 83 (1999), 3398-3401.

[6] David Hunger, Stephan Camerer, Theodor W. Hänsch, Daniel König, Jörg P. Kotthaus, Jakob Reichel and Philipp Treutlein, Phys. Rev. Lett., 104 (2010), 143002.

[7] Y. Lin, I. Teper, C. Chin and V. Vuletić, Phys. Rev. Lett., 92 (2004), 050404.

[8] D. M. Harber, J. M. Obrecht, J. M. McGuirk and E. A. Cornell, Phys. Rev. A, 72 (2005), 033610.

[9] Richard J. Cook and Richard K. Hill, Optics Communications, 43 (1982), 258-260.

[10] J. P. Dowling and J. GeaBanacloche, Adv. Atom. Mol. Opt. Phys., 37 (1996), 1-94.

[11] Hammes, M. and Rychtarik, D. and Engeser, B. and Nägerl, H.-C. and Grimm, R., 90 (2003), 173001.

[12] Fam Le Kien, V. I. Balykin and K. Hakuta, Phys. Rev. A, 70 (2004), 063403.

[13] E. Vetsch, D. Reitz, G. Sague, R. Schmidt, S.T. Dawkins and A. Rauschenbeutel, Phys. Rev. Lett., 104 (2010), 203603.

[14] A. Goban, K. S. Choi, D. J. Alton, D. Ding, C. Lacroute, M. Pototschnig, T. Thiele, N. P. Stern and H. J. Kimble, Phys. Rev. Lett., 109 (2012), 033603.

[15] R. A. Cornelussen, A. H. van Amerongen, B. T. Wolschrijn, R. J. C. Spreeuw and H. B. V. van den Heuvell, Eur. Phys. J. D, 21 (2002), 347-351.

[16] J. I. Gillen, W. S. Bakr, A. Peng, P. Unterwaditzer, S. Fölling and M. Greiner, Phys. Rev. A, 80 (2009), 021602.

[17] M. Miranda, A. Nakamoto, Y. Okuyama, A. Noguchi, M. Ueda and M. Kozuma, Phys. Rev. A, 86 (2012), 063615.

[18] V. Sandoghdar, C. I. Sukenik, E. A. Hinds, and Serge Haroche, Phys. Rev. Lett. 68 (1992), 34323435.

[19] Takao Aoki, Barak Dayan, E. Wilcut, W.P. Bowen, A. S. Parkins, T. J. Kippenberg, K. J. Vahala and H. J. Kimble, Nature, 443 (2006), 671-674.

[20] N. Schlosser, G. Reymond, I. Protsenko and P. Grangier, Nature, 411 (2001), 1024-1027.

[21] N. Schlosser, G. Reymond and P. Grangier, Phys. Rev. Lett., 89 (2002), 023005.

[22] J. D. Thompson, T. G. Tiecke, A. S. Zibrov, V. Vuletić and M. D. Lukin, arXiv:1209.3028.

[23] J. R. Wait, Can. J. Phys., 33 (1955), 189-195.

[24] M. T. Homer Reid, http://homerreid.ath.cx/scuff-em/, (2012).

[25] N. P. Stern, D. J. Alton and H. J. Kimble, New J. Phys. 13 (2011), 085004.

[26] Lord Rayleigh, Phil. Mag., xii (1881), 81.

[27] Linyou Cao, Justin S. White, Joon-Shik Park, Jon A. Schuller, Bruce M. Clemens and Mark L. Brongersma, Nat. Mater., 8 (2009), 643-647.

[28] C. Lacroute, K. S. Choi, A. Goban, D. J. Alton, D. Ding, N. P. Stern and H. J. Kimble, New J. Phys., 14 (2012), 023056. 


\section{ICAP 2012}

[29] J. D. Thompson, et. al, in preparation.

[30] Silvia Bergamini, Benoît Darquié, Matthew Jones, Lionel Jacubowiez, Antoine Browaeys and Philippe Grangier, J. Opt. Soc. Am. B, 21 (2004), 1889-1894.

[31] Savas Dimopoulos and Andrew A. Geraci, Phys. Rev. D, 68 (2003), 124021.

[32] M. Gullans, T. G. Tiecke, D. E. Chang, J. Feist, J. D. Thompson, J. I. Cirac, P. Zoller, M. D. Lukin, Phys. Rev. Lett., 109 (2012), 235309.

[33] H. J. Kimble, Nature, 453 (2008), 1023-1030. 